\title{
Three-dimensional modeling and numerical analysis of fractional flow reserve in human coronary arteries
}

\author{
Neng Dai ${ }^{1}$, Hui-Jie Lv², Ya-Fei Xiang ${ }^{2}$, Bing Fan ${ }^{3}$, Wei-Ming $\mathrm{Li}^{1}$, Ya-Wei Xu \\ ${ }^{1}$ Cardiology Department, Tenth People's Hospital, Tongji University School of Medicine, Shanghai, China \\ 2Department of Mathematics, Tongji University, Shanghai, China \\ ${ }^{3}$ Cardiology Department, Zhongshan Hospital, Fudan University, Shanghai, China
}

Adv Interv Cardiol 2016; 12, 1 (43): 25-31

DOI: $10.5114 /$ pwki.2016.56946

\begin{abstract}
A bstract
Introduction: Noninvasive fractional flow reserve (FFR) computed from CT (FFR ${ }_{C T}$ ) is a novel method for determining the physiologic significance of coronary artery disease (CAD). Several clinical trials have been conducted, but its diagnostic performance varied among different trials.

Aim: To determine the cut-off value of $\mathrm{FFR}_{\mathrm{CT}}$ and its correlation with the gold standard used to diagnose CAD in clinical practice.

Material and methods: Forty patients with single vessel disease were included in our study. Computed tomography scan and coronary angiography with FFR were conducted for these patients. Three-dimensional geometric reconstruction and numerical analysis based on the computed tomographic angiogram (CTA) of coronary arteries were applied to obtain the values of FFR ${ }_{C T}$ The correlation between $\mathrm{FFR}_{\mathrm{CT}}$ and the gold standard used in clinical practice was tested.

Results: For FFR $\mathrm{CT}_{\mathrm{C}}$ the best cut-off value was 0.76 , with the sensitivity, specificity, positive predictive value and negative predictive values of $84.6 \%, 92.9 \%, 88 \%$ and $73.3 \%$, respectively. The area under the receiver-operator characteristics curve was 0.945 $(p<0.0001)$. There was a good correlation of FFR $\mathrm{CT}_{\mathrm{CT}}$ values with FFR values $(r=0.94, p<0.0001)$, with a slight overestimation of FFR as compared with measured FFR (mean difference $0.01 \pm 0.11, p<0.05$ ). For inter-observer agreement, the mean $\kappa$ value was 0.69 (0.61 to 0.78 ) and for intra-observer agreement the mean $\kappa$ value was 0.61 (0.50 to 0.72 ).

Conclusions: $F_{F R}$ derived from CT of the coronary artery is a reliable non-invasive way providing reliable functional information of coronary artery stenosis.
\end{abstract}

Key words: fractional flow reserve, cardiac imaging, non-invasive fractional flow reserve.

\section{Introduction}

Coronary artery disease is more and more common in developed and developing countries, and has an important impact on the morbidity and mortality statistics and health economics worldwide [1, 2]. Diagnosis of coronary artery disease is important in risk stratification and guides further management. Invasive coronary angiography is the traditional method of imaging the coronary arteries and remains the gold standard. It detects luminal stenosis but provides little information about the vessel wall or plaques [3]. Besides, not all anatomical lesions are functionally significant. This has led to a wide variety of imaging techniques to identify and assess a flow-limiting stenosis. The approach to diagnosis of coronary artery disease is broadly based on anatomical and functional imaging [4].

Fractional flow reserve derived by computed tomography $\left(F F R_{C T}\right)$ is an emerging noninvasive technique that could evaluate the functional severity of coronary artery stenoses. FFR $\mathrm{CT}_{\mathrm{CT}}$ is defined as the fraction of distal pressure of coronary artery stenoses to that of proximal pressure, which is calculated in three-dimensional models of the coronary tree. Several recent clinical trials - De-FACTO [5], DISCOVER-FLOW [6] and NXT [7] have validated this new cardiac imaging modality and evaluated its diagnostic performance. However, the cornerstone of $\mathrm{FFR}_{\mathrm{CT}}$ is the linear relationship between pressure and flow under conditions of constant (and minimized) intracoronary resistance, as the situation

Corresponding author:

Dr. Ya-Wei Xu, Dr. Wei-Ming Li, Cardiology Department, Tenth People’s Hospital, Tongji University School of Medicine, No. 301 Middle Yanchang Road, 200072 Shanghai, China, phone: +860216630 0588, fax: +860216630 0588, e-mail: niceday1987@hotmail.com, 18917683469@189.cn

Received: 9.03.2015, accepted: 10.08.2015. 
achieved in the measurement of invasive FFR, since they have the same formula and theory for calculation [8]. In clinical practice, physicians usually use adenosine to achieve hyperemia during FFR measurement. In these three main trials investigating $\mathrm{FFR}_{\mathrm{CT}}$, the authors used computer stimulated hyperemia instead of adenosine during acquisition of the computed tomography (CT) image, which is different from that of the real situation and would affect the results. Also the cut-off value of 0.80 was applied for FFR ${ }_{C T}$ which seems arbitrary. Moreover, the diagnostic performance of $F_{C R}$ is not uniform [9] among the three trials, leaving space for improvement of this index.

\section{Aim}

Thus, we conducted the present study to determine the cut-off value of $\mathrm{FFR}_{\mathrm{CT}}$ and re-assess its diagnostic performance, during which adenosine triphosphate (ATP) was used to stimulate hyperemia before the CT scan.

\section{Material and methods}

\section{Study population}

To be included, patients had to be adults with single-vessel disease who underwent invasive coronary angiography after computed tomography angiogram (CTA). Exclusion criteria were: a history of coronary artery bypass surgery (CABG); significant arrhythmia; heart rate $>100$ beats/min; systolic blood pressure < $90 \mathrm{~mm} \mathrm{Hg}$; non-cardiac illness with life expectancy $<2$ years; pregnant state; serum creatinine $\geq 1.7 \mathrm{mg} / \mathrm{dl}$; allergy to iodinated contrast; contraindication to $\beta$-blockers, nitroglycerin or ATP; and unstable coronary heart disease. The study protocols were approved by the Tongji University ethics committee. Written informed consent to participate was obtained from all patients eligible.

\section{CT scanning}

Coronary CT was performed in accordance with Society of Cardiovascular Computed Tomography guidelines [10]. ATP was used for hyperemia via the large antecubital vein during CT scanning (140 mg/kg/min). Oral and/or i.v. $\beta$-blockers were used to achieve a heart rate $<60$ beats $/$ min. Assessment of luminal diameter stenosis was performed at the discretion of the local investigator using an 18-segment coronary model. Significant coronary obstruction was defied as stenosis severity $>50 \%$ in a major coronary artery segment $2 \mathrm{~mm}$ in diameter.

\section{FFR measurement}

Invasive coronary angiography (ICA) was performed according to a standard protocol [11]. Intracoronary nitroglycerine was administered before FFR measurements. The FFR measurement was performed for the vessels with stenosis (PressureWire; St. Jude Medical, St.
Paul, Minnesota). Coronary hyperemia was induced by the administration of intravenous ATP $(140 \mathrm{mg} / \mathrm{kg} / \mathrm{min})$. Intracoronary pressure was measured after 2 min of adenosine infusion. 0.80 was selected as the cut-off value for FFR.

\section{Three-dimensional geometric model}

Firstly, a sequence of CT scan images of the coronary artery was collected from the Dual Source Computed Tomography scanner (Somatom Sensation and Definition $\mathrm{CT}$, Siemens, Forchheim, Germany). The images, which were in DICOM format, were of the size $512 \times 512 \mathrm{~mm}$ and the resolution $0.869 \mathrm{~mm}$. $269 \mathrm{CT}$ images in total for each patient were collected and the slice increment was $1.250 \mathrm{~mm}$.

Secondly, we separated coronary arteries and their branch vessels from myocardium and partial aorta with a progressive region growing technique [12]. The resulting binary segmentation mask enclosed the vessel lumen, as well as potential plaques. The segmentation mask was converted into a surface mesh using a marching cube algorithm. This mesh, which surrounds the vessel lumen and plaques, is regarded to represent the lumen of the artery in its healthy state. The plaque was manually segmented by applying a region growing technique: starting from a seed point within the plaque, all connected voxels with intensity values greater than a threshold value were regarded as representing the plaque. This threshold was determined to be 516 Hounsfield units by visual inspection of the segmentation results superimposed on the original data set. All voxels belonging to the plaque were then removed from the segmentation mask, and a surface mesh enclosing the artery lumen without plaque was constructed. This mesh is regarded to represent the lumen of the artery in its diseased state. After these operations, we constructed a 3D model of the coronary arteries for our analysis. One of the reconstructed coronary arteries is shown in Figure 1.

\section{Mathematical model}

First, we chose a diseased coronary artery that had a lesion of moderate severity to study the model. In the process of studying the blood dynamics, we create a simplified mathematical model. We took the coronary artery as an inflexible pipe, since the coronary arteries had attained hyperemia by administration of intravenous (140 $\mathrm{kg} / \mathrm{kg} / \mathrm{min})$ ATP before CTA image acquisition; thus, the deformation of the coronary artery with pulse blood can be neglected.

Then we considered several parameters to simulate blood flow in the coronary artery. There are a number of fundamental assumptions about coronary blood flow used when deriving the governing equations for the model presented here. The studies of Perktold et al. [13] and Cho and Kensey [14] indicate no significant 

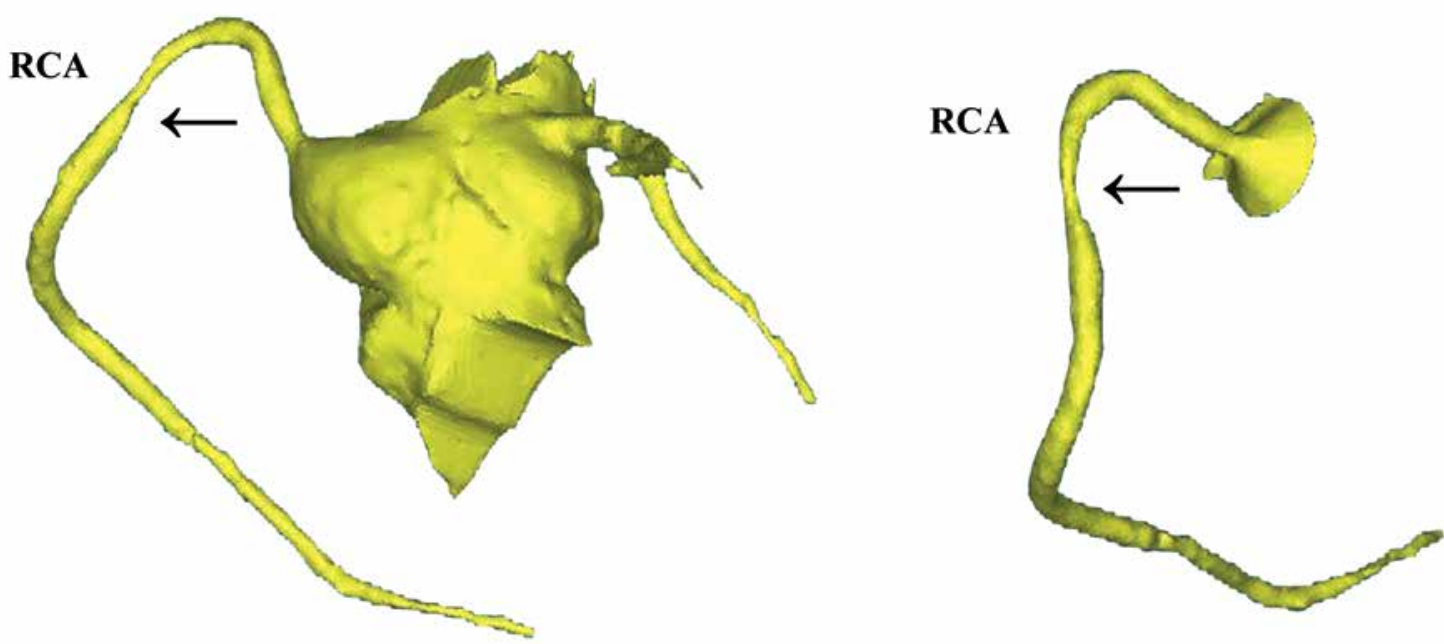

Figure 1. Reconstructed image of right coronary artery in one of the included patients. Three-dimensional modeling of right coronary artery for one of the included patients. The black arrow indicates an intermediate stenosis in the proximal part of the right coronary artery

RCA - Right coronary artery.

influence of the shear thinning properties of blood in large vessels, and thus blood viscosity is assumed constant and independent of vessel radius. To account for the nonuniform distribution of red blood cells over the cross section of narrower blood vessels, blood can be modeled as a continuum. The distensibility of a coronary vessel wall is assumed to dominate any effects due to the compressibility of blood.

In summary, blood flow in coronary arteries can be taken as a homogeneous and incompressible viscous Newtonian fluid, given by:

$\nabla \cdot U=0$

$\rho \frac{\partial U}{\partial t}+\rho(U \cdot \nabla) U=-\nabla p+\mu \nabla^{2} U$

Blood flow in coronary arteries is laminar because the Reynolds number $[15,16], R_{e} \leq 2000$, defined as $R_{e}=$ $\rho V D / \mu$, where $\rho$ is the density of fluid, $V$ is the average velocity of fluid, $D$ is the inner diameter of the pipe and $\mu$ is the dynamic viscosity of the fluid. According to fluid mechanics, the flow is laminar when $R_{e} \leq 2000$, while it is turbulent when $R_{e} \geq 3000$.

Next, we discuss the average velocity of blood flow in the coronary artery, namely $V=C / \pi R^{2}$.

In this equation, $C$ is the output of blood from the aorta every second and $R$ is the radius of the coronary vessel, then the Reynolds number of blood can be computed by $R_{e}=2 C \rho / \pi R \mu$. Taking the average volume of blood flow (the average volume of blood flow in the left coronary artery is $0.75 \mathrm{ml}$, while in the right it is $0.25 \mathrm{ml}$ ) and the heart rate is 75 times per minute, the Reynolds number $R_{e}=72<2000$ or $R_{e}=24<2000$. It follows that the blood flow in the coronary artery is laminar at the entrance, though it may be disturbed in the stenotic or bifurcated area [17].

The viscosity and the density of blood are $\mu=3.5 \times$ $10^{-3} \mathrm{~Pa} \cdot \mathrm{s}$ and $\rho=1050 \mathrm{~kg} \cdot \mathrm{m}^{-3}$ respectively, which is to mimic the blood properties in large epicardial arteries.

\section{Finite element method}

According to the three-dimensional geometric model, the finite element method was used to simulate and analyze the velocity and pressure of blood flow in the coronary artery [18-20]. The FLOTRAN 142 element was chosen in the software ANSYS 13.0 to compute numerical results. The total number of nodes was 95,099 and the number of elements was 1,040,787.

The initial and boundary conditions of the blood flow are taken as follows: both the velocity on the arterial wall and the pressure on outlet boundaries are 0; the inlet velocity $V_{\text {inlet } \Psi}(\mathrm{cm} / \mathrm{s})$ is 6.

\section{Statistical analysis}

All statistical analyses were performed using MedCalc for Windows (Version 12.5; Ostend, Belgium). Continuous variables were presented as means \pm SDs, and ordinal variables were presented as medians with interquartile ranges. Statistical significance was considered when a $p$ value $<0.05$ was observed. Our primary results were the diagnostic performance of $F_{F R}$ using invasive FFR as the reference, including its sensitivity, specificity, positive predictive value, and negative predictive value. In addition, the best cut-off value of FFR ${ }_{C T}$ to detect functional CAD was determined by the receiver operat- 
ing characteristics curve (ROC). An analysis according to Bland-Altman was performed [21]. Pearson statistics was applied to analyze the degree of correlation between FFR derived by CTA and the invasive one. Moreover, inter- and intra-observer variability was assessed by $\kappa$ analysis [22]. A $\kappa$ coefficient value of less than 0.00 suggests poor agreement, 0.00 to 0.20 slight agreement, 0.21 to 0.40 fair, 0.41 to 0.60 moderate, 0.61 to 0.80 substantial and 0.81 to 1.00 almost perfect agreement.

\section{Results}

\section{Patients' characteristics and statistical analysis}

We included 40 patients (13 males, 27 females) who were admitted to the Cardiology department, Tenth People's Hospital, Tongji University School of Medicine for analysis. As shown in Table I, the average age was $63.7 \pm 9.4$ years. All of them had moderate lesions ( $50 \%$ to $70 \%$ ) in a single artery (12 right coronary arteries, 7 left main arteries, 17 left anterior descending arteries, 4 left circumflex arteries). The mean analysis time for each case was $224 \pm 31 \mathrm{~min}$.

Table I. Baseline characteristics of study population $(n=40)$

\begin{tabular}{|c|c|}
\hline Parameter & Result \\
\hline Age [years] & $63.7 \pm 9.4$ \\
\hline Male & $13(32.5 \%)$ \\
\hline Body mass index $\left[\mathrm{kg} / \mathrm{m}^{2}\right]$ & $28.8 \pm 4.7$ \\
\hline \multicolumn{2}{|l|}{ Cardiovascular risk factors: } \\
\hline Hypertension & $23(57.5 \%)$ \\
\hline Diabetes mellitus & $14(35 \%)$ \\
\hline Dyslipidemia & $31(77.5 \%)$ \\
\hline Smoking & $7(17.5 \%)$ \\
\hline \multicolumn{2}{|l|}{ Laboratory measures: } \\
\hline Glycated hemoglobin (\%) & $6.1 \pm 2.6$ \\
\hline Triglycerides [mmol/l] & $142.5 \pm 97.3$ \\
\hline Total cholesterol [mg/dl] & $210.2 \pm 49.1$ \\
\hline HDL cholesterol [mg/dl] & $47.7 \pm 12.4$ \\
\hline Creatinine $[\mathrm{mg} / \mathrm{dl}]$ & $0.9 \pm 0.2$ \\
\hline \multicolumn{2}{|l|}{ Vessels with lesions: } \\
\hline$L M$ & 7 \\
\hline LAD & 17 \\
\hline$\overline{L C X}$ & 4 \\
\hline RCA & 12 \\
\hline
\end{tabular}

$H D L$ - High-density lipoprotein, $L A D$ - left anterior descending artery, $L C X$ - left circumflex artery, LM - left main artery, RCA - right coronary artery.
As seen in Figure 2, the best cut-off value for FFR $_{C T}$ seems to be 0.76 , with the sensitivity, specificity, positive predictive value, and negative predictive value as $84.6 \%$, $92.9 \%, 88 \%, 73.3 \%$, respectively. The area under the receiver-operator characteristics curve was 0.945 ( $p<0.0001$ ).

There is a good correlation of $F_{F R}$ values with FFR values (Pearson's correlation coefficient $=0.94, p<$ 0.001), with a slight overestimation of $F_{F R}$ as compared with measured FFR (mean difference $0.01 \pm 0.11$, $p<0.05$ ) (Figures 3,4). Data of one patient in our study population are shown in Figures 5 and 6.

For inter-observer agreement, the mean $\kappa$ value was 0.69 (0.61 to 0.78) and for intra-observer agreement the mean $\kappa$ value was 0.61 (0.50 to 0.72 ).

\section{Discussion}

In clinical practice, patients presenting with chest pain are common [23]. In these cases, physicians must determine whether the patients have coronary artery disease and, if so, whether the patients are at increased risk of future cardiovascular disease. Current guidelines recommend that this evaluation should be differential based on patient pretest risk assessment. Low-risk patients should receive only expectant management, intermediate-risk patients should be referred for noninvasive testing, and high-risk patients should undergo invasive cardiac catheterization [24]. However, to date, noninvasive tests have performed this evaluation by taking either an anatomic approach such as CT angiograms to identify obstructive CAD [25] or a functional approach to determine ischemia by perfusion or wall motion function via

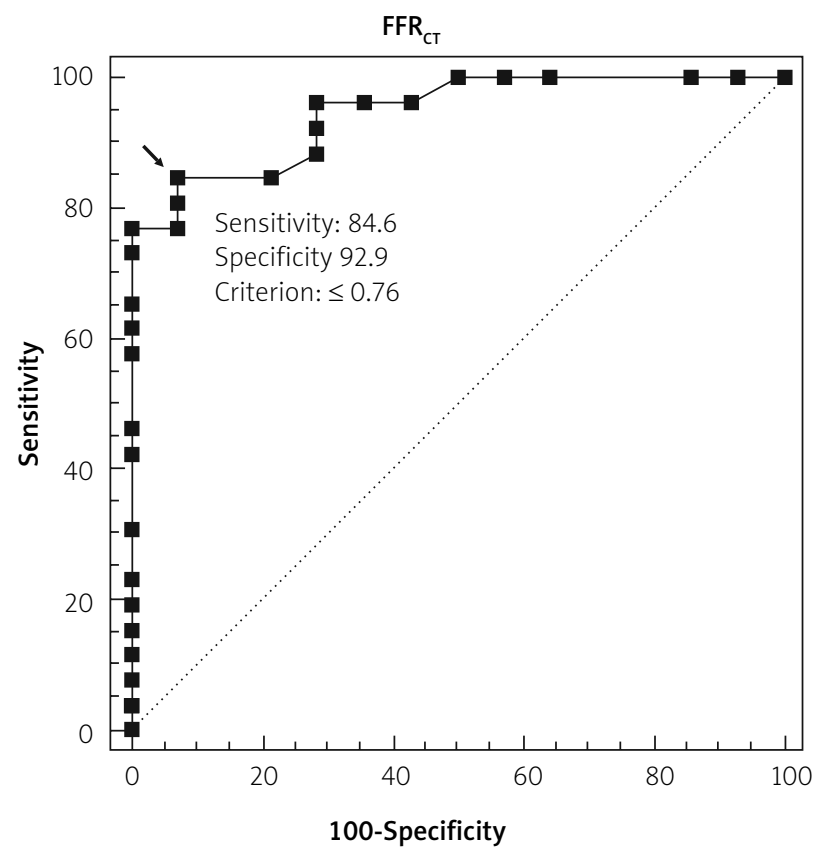

Figure 2. ROC curve for FFR ${ }_{C T}$ As shown in the ROC curve, 0.76 may be the best cut-off value for FFR (sensitivity $=84.6 \%$, specificity $=92.9 \%$ ) 


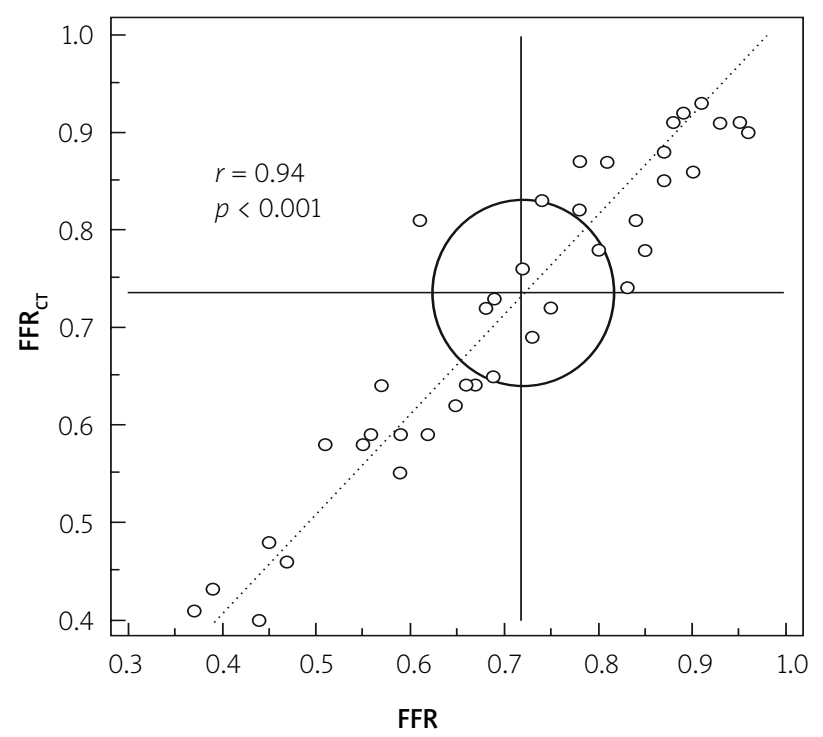

Figure 3. Correlation between $F F R_{C T}$ and FFR. A good correlation of $F_{F R}$ to FFR is observed (Pearson correlation coefficient $r=0.94, p<0.001$ )

a variety of stress and imaging modes [26]. And for patients with moderate stenoses, risk classification is really a challenge for physicians $[27,28]$, though coronary angiography is widely accepted as the gold standard investigation to diagnose coronary artery disease. The catheter (typically no more than $2.0 \mathrm{~mm}$ in diameter) placed in the artery to create the $\mathrm{X}$-ray images could lead to secondary damage to coronary arteries. What is more, it is highly subjective and does not provide any information about the hemodynamics of stenosis. Therefore, technologies that provide both a highly sensitive anatomic evaluation for obstructive disease and a highly specific physiologic evaluation for ischemia are needed for noninvasive imaging for CAD. In our study, computational fluid dynamics was applied to calculate $\mathrm{FFR}_{\mathrm{CT}}$ It is a new, non-invasive measurement of not only the structural but also the functional severity of coronary artery stenoses. Compared to angiography, our three-dimensional model constructed by CT images is non-invasive and based on computer analysis of the images. It takes just a little time to present coronary arteries by construction of a three dimensional model of coronary arteries in the computer, then to calculate $F_{F R}$ in the reconstructed coronary arteries.

The diagnostic value of $F F R_{C T}$ has been evaluated in several previous studies, but simulative hyperemia was applied in previous studies, which may affect the result because hyperemia is the most important precondition for FFR measurement.

In our study, inter- and intra-observer agreement were substantial with $\kappa$ coefficient values of 0.69 and 0.61 , respectively, thus indicating that our model used for $F_{C T}$ calculation is robust and reproducible.

To our knowledge, our study used ATP to induce hyperemia during CT scanning for the first time and our

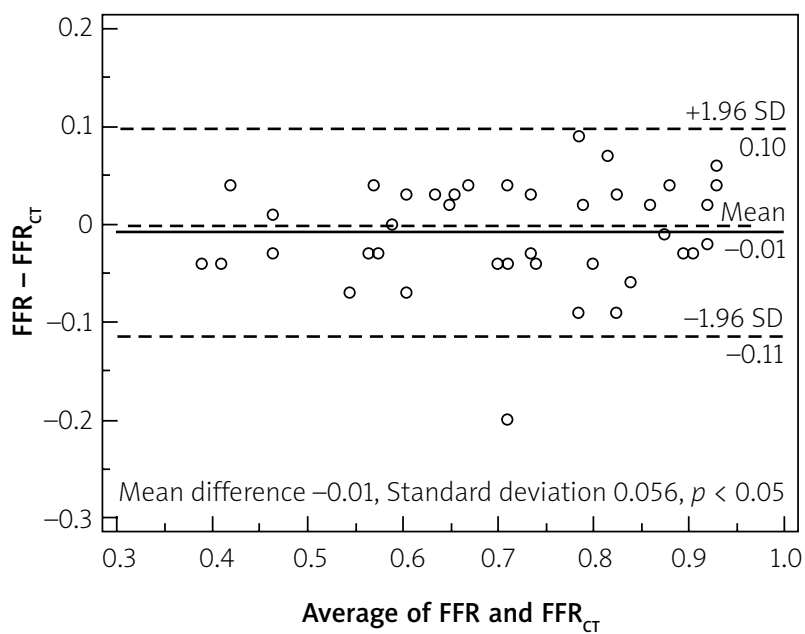

Figure 4. Bland-Altman plot of FFR and $F_{F R}$ A slight systematic overestimation of computation of fractional flow reserve from $F_{F R}$ as compared with FFR is observed (mean difference: -0.01 , standard deviation: $0.11, p<0.05$ )

results reveal that the value of $F_{F R}$ is in good correlation with that obtained from invasive FFR. Thus, we can use the $\mathrm{FFR}_{\mathrm{CT}}$ as a diagnostic tool in patients with moderate lesions in their coronary arteries to choose the suitable therapy. For FFR, the cut-off value is 0.80 [29], while the best cut-off value for $F_{C R}$ is 0.76 according to the ROC curve (Figure 2). This is in accordance with a previous study [30]. That is to say, stenoses with an FFR $\mathrm{FT}_{\mathrm{CT}}$ measurement of $<0.76$ are almost invariably able to induce myocardial ischemia and further invasive examinations such as angiography are needed for these patients, while stenoses with $\mathrm{FFR}_{\mathrm{CT}} \geq 0.76$ may not be responsible for cardiac ischemia. However, the prognostic value of $F_{F R}$ in CAD remains to be studied.

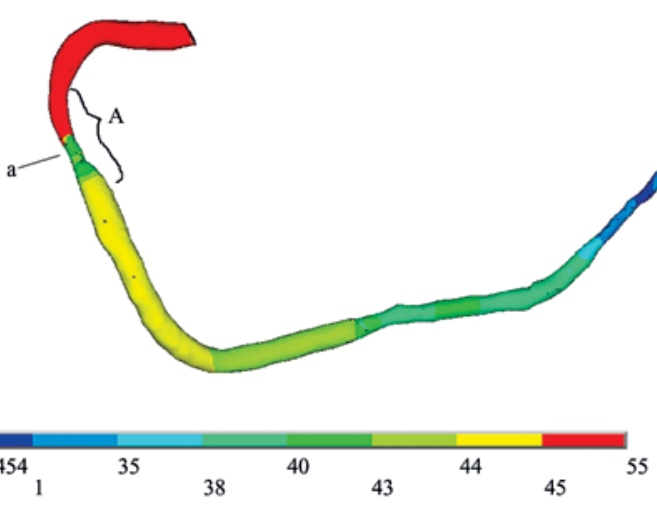

Figure 5. FFR $\mathrm{CT}_{\mathrm{C}}$ derived from the CTA in the same patient as Figure 1. Distribution of blood pressure in the reconstructed right coronary artery as in Figure 1 was analyzed and the value of FFR $\mathrm{FT}_{\mathrm{CT}}$ was 0.82 for the lesion in this patient 


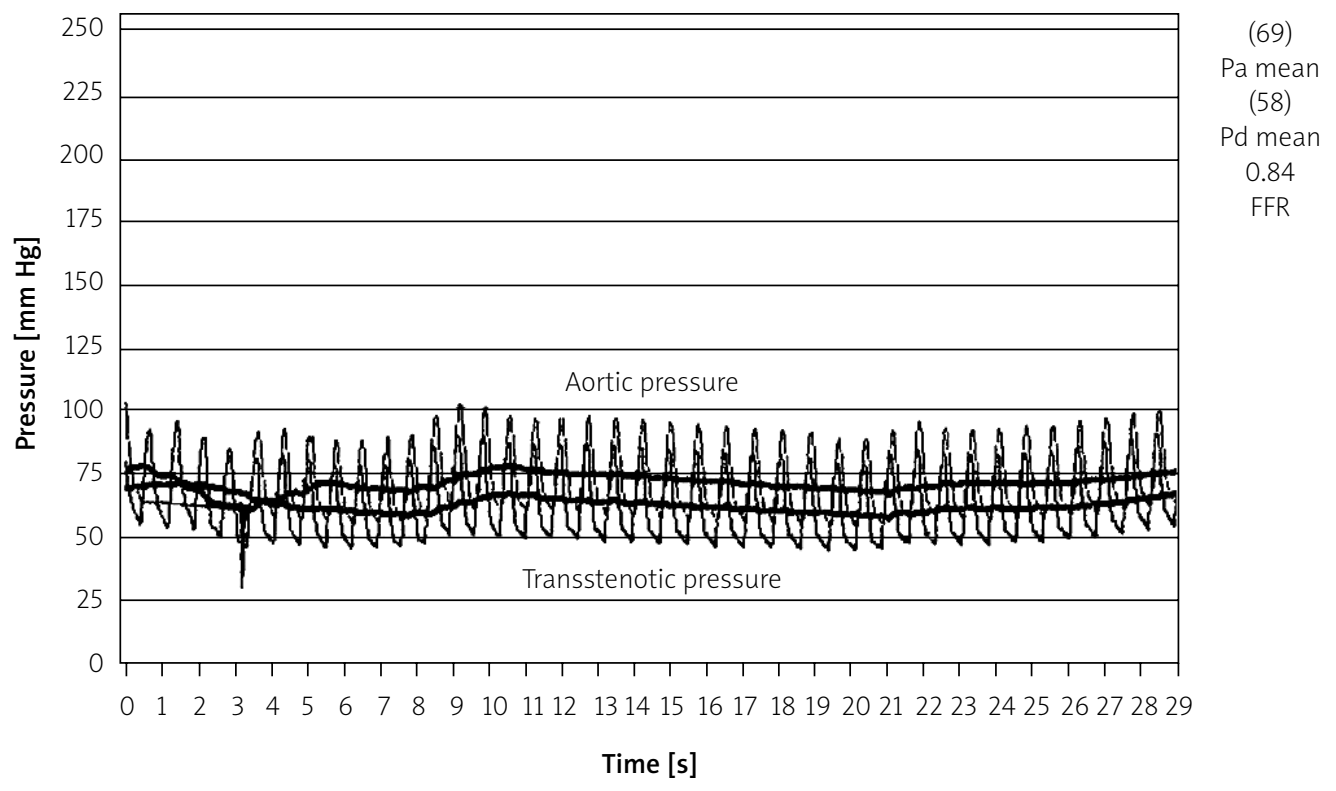

Figure 6. Invasive FFR measurement for the same patient as Figure 1 . The value of invasive FFR is 0.84 for the lesion in the same patient as Figure 1 , nearly equal to the value of FFR ${ }_{C T}$, which indicated that the stenosis was not functionally important

There were also some limitations in our study. First, the coronary arteries were reconstructed from the CT data. We actually did not have every lay of the coronary artery during the CT scan, so the reconstructed coronary arteries are not strictly same as the real-life ones. Second, in human coronary arteries, vessel walls are elastic and undergo a considerable degree of geometric deformation during each cardiac cycle due to the contraction and relaxation of cardiac muscle. However, because most blood flow in coronary arteries occurs during the diastolic period of the cardiac cycle when both the heart and coronary arteries are fairly well inflated and stretched close to their maximum sizes and ATP was used to achieve hyperemia during CT images acquisition, it was assumed that the flow patterns in coronary arteries are not affected significantly by the elastic nature of the vessel wall. Finally, we only included patients with single coronary artery stenosis. The diagnostic performance of FFR $\mathrm{CT}_{\mathrm{CT}}$ needs to be tested in a wider spectrum of diseases such as multivessel coronary artery disease, diffuse and serial diseased lesions, bifurcation lesions, in-stent restenosis, etc., before it can finally enter clinical application.

\section{Conclusions}

$\mathrm{FFR}_{\mathrm{CT}}$ derived from CT of the coronary artery is a reliable, non-invasive way of providing reliable functional information of coronary artery stenosis.

\section{Acknowledgments}

This study was funded, in part, by the Country China Scholarship Council and was funded by the Medical
Related Science and Technology Project of Shanghai Science and Technology Commission (No. 14411963100).

\section{Conflict of interest}

The authors declare no conflict of interest.

\section{References}

1. Rajadurai J, Arokiasamy J, Pasamanickam K, et al. Coronary artery disease in Asians. Aust N Z J Med 1992; 22: 345-8.

2. Centers for Disease Control and Prevention (CDC). Prevalence of coronary heart disease--United States, 2006-2010. MMWR Morb Mortal Wkly Rep 2011; 60: 1377-81.

3. Henry TD, Sharkey SW. How gold is the gold standard? How gold does it need to be? Catheter Cardiovasc Interv 2012; 79: 1099100.

4. Al-Hassan D, Leipsic J. Noninvasive fractional flow reserve derived from coronary computed tomography angiography: integrated anatomical and functional assessment. Future Cardiol 2013; 9: 243-51.

5. Min JK, Leipsic J, Pencina MJ, et al. Diagnostic accuracy of fractional flow reserve from anatomic CT angiography. JAMA 2012; 308: 1237-45.

6. Koo BK, Erglis A, Doh JH, et al. Diagnosis of ischemia-causing coronary stenoses by noninvasive fractional flow reserve computed from coronary computed tomographic angiograms. Results from the prospective multicenter DISCOVER-FLOW (Diagnosis of Ischemia-Causing Stenoses Obtained Via Noninvasive Fractional Flow Reserve) study. J Am Coll Cardiol 2011; 58: 1989-97.

7. Nørgaard BL, Leipsic J, Gaur S, et al. Diagnostic performance of noninvasive fractional flow reserve derived from coronary computed tomography angiography in suspected coronary artery disease: the NXT trial (Analysis of Coronary Blood Flow Using CT Angiography: Next Steps). J Am Coll Cardiol 2014; 63: 1145-55. 
8. Pijls NH, Van Gelder B, Van der Voort P, et al. Fractional flow reserve. A useful index to evaluate the influence of an epicardial coronary stenosis on myocardial blood flow. Circulation 1995; 92: 3183-93.

9. Dai N, Xia HH, Xu YW. Noninvasive approach to assess coronary artery stenoses and ischemia. JAMA 2013; 309: 235-6.

10. Abbara S, Arbab-Zadeh A, Callister TQ, et al. SCCT guidelines for performance of coronary computed tomographic angiography: a report of the Society of Cardiovascular Computed Tomography Guidelines Committee. J Cardiovasc Comput Tomogr 2009; 3: 190-204.

11. Naidu SS, Rao SV, Blankenship J, et al. Clinical expert consensus statement on best practices in the cardiac catheterization laboratory: society for cardiovascular angiography and interventions. Catheter Cardiovasc Interv 2012; 80: 456-64.

12. William EL, Harvey EC. Marching cubes: a high resolution 3D surface construction algorithm. Comput Graph (ACM) 1987; 21: 163-9.

13. Perktold K, Resch M, Peter RO. Three-dimensional numerical analysis of pulsatile flow and wall shear stress in the carotid artery bifurcation. J Biomech 1991; 24: 409-20.

14. Cho YI, Kensey KR. Effects of the non-Newtonian viscosity of blood on flows in a diseased arterial vessel. Part 1: steady flows. Biorheology 1991; 28: 241-62.

15. Chatzizisis YS, Coskun AU, Jonas $M$, et al. Role of endothelial shear stress in the natural history of coronary atherosclerosis and vascular remodeling: molecular, cellular, and vascular behavior. J Am Coll Cardiol 2007; 49: 2379-93.

16. Back LD, Radbill JR, Crawford DW. Analysis of pulsatile, viscous blood flow through diseased coronary arteries of man. J Biomech 1977; 10: 339-53.

17. Asakura T, Karino T. Flow patterns and spatial distribution of atherosclerotic lesions in human coronary arteries. Circ Res 1990; 66: 1045-66.

18. Wang KC, Dutton RW, Taylor CA. Improving geometric model construction for blood flow modeling. IEEE Eng Med Biol Mag 1999; 18: 33-9.

19. Bathe M, Kamm RD. A fluid: structure interaction finite element analysis of pulsatile blood flow through a compliant stenotic artery. J Biomech Eng 1999; 121: 361-9.

20. Müller J, Sahni O, Li X, et al. Anisotropic adaptive finite element method for modelling blood flow. Comput Methods Biomech Biomed Engin 2005; 8: 295-305.

21. Bland JM, Altman DG. Statistical methods for assessing agreement between two methods of clinical measurement. Lancet 1986; 1: 307-10.

22. Fleiss JL, Levin B, Paik MC. Statistical methods for rates and proportions. 3rd ed. John Wiley \& Sons, Hoboken 2003.

23. Ruigómez A, Rodríguez LA, Wallander MA, et al. Chest pain in general practice: incidence, comorbidity and mortality. Fam Pract 2006; 23: 167-74.

24. Fraker TD Jr, Fihn SD, et al. 2007 chronic angina focused update of the ACC/AHA 2002 guidelines for the management of patients with chronic stable angina: a report of the American College of Cardiology/American Heart Association Task Force on Practice Guidelines Writing Group to develop the focused update of the 2002 guidelines for the management of patients with chronic stable angina. J Am Coll Cardiol 2007; 50: 2264-74.

25. Goldstein JA, Chinnaiyan KM, Abidov A, et al. The CT-STAT (Coronary Computed Tomographic Angiography for Systematic Triage of Acute Chest Pain Patients to Treatment) trial. J Am Coll Cardiol 2011; 58: 1414-22.

26. Nelson KH, Willens HJ, Hendel RC. Utilization of radionuclide myocardial perfusion imaging in two health care systems: assessment with the 2009 ACCF/ASNC/AHA appropriateness use criteria. J Nucl Cardiol 2012; 19: 37-42.

27. Pijls NH, De Bruyne B, Peels K, et al. Measurement of fractional flow reserve to assess the functional severity of coronary-artery stenoses. N Engl J Med 1996; 334: 1703-8.

28. Fard-Esfahani A, Assadi M, Saghari M, et al. The role of myocardial perfusion imaging in the evaluation of patients undergoing percutaneous transluminal coronary angioplasty. Hellenic J Cardiol 2009; 50: 396-401.

29. Pijls NH, Van Gelder B, Van der Voort P, et al. A useful index to evaluate the influence of an epicardial coronary stenosis on myocardial blood flow. Circulation 1995; 92: 3183-93.

30. Kim KH, Doh JH, Koo BK, et al. A novel noninvasive technology for treatment planning using virtual coronary stenting and computed tomography-derived computed fractional flow reserve. JACC Cardiovasc Interv 2014; 7: 72-8. 